\title{
Malignant Lacrimal Gland Neoplasm
}

National Cancer Institute

\section{Source}

National Cancer Institute. Malignant Lacrimal Gland Neoplasm. NCI Thesaurus. Code C3563.

Primary or metastatic malignant neoplasm affecting the lacrimal gland. 